\title{
Utilização do Ambiente WebAPSEE na implantação do nível G do MPS.BR no CTIC-UFPA
}

\author{
Breno B. N. de França, Ernani de O. Sales, Carla A. L. Reis, Rodrigo Quites Reis
}

Programa de Pós-Graduação em Ciência da Computação - Instituto de Ciências Exatas e Naturais - Universidade Federal do Pará (UFPA) - Belém - PA - Brasil

Laboratório de Engenharia de Software - Universidade Federal do Pará (UFPA)

$\{$ breno, ernani, clima, quites\} @webapsee.com

\begin{abstract}
WebAPSEE environment is one of the current alternatives to support Software Process Management concerning current standards and quality models. This paper shows a successful experience about the use of this PSEE on the MPS.BR level G implementation at CTIC-UFPA. The observed positive aspects and improvement opportunities are related as well as some lessons learned.
\end{abstract}

Resumo. $O$ ambiente WebAPSEE é uma das alternativas atuais para automatizar a gerência de processos de software com objetivo de atender padrões e modelos de qualidade existentes. Este artigo apresenta uma experiência de sucesso na utilização dessa ferramenta na implementação do nível $G$ do MPS.BR no CTIC-UFPA. Os pontos positivos e as oportunidades de melhoria do uso desse ambiente bem como as lições aprendidas nessa experiência são relatados.

\section{Introdução}

A melhoria de processos de software em empresas brasileiras tem ocorrido cada vez mais em consonância com o modelo de Melhoria do Processo de Software Brasileiro (MPS.BR) [Softex 2007]. Com mais de 120 empresas avaliadas desde a sua criação em 2003, esse modelo tem cumprido as suas metas estabelecidas e contribuído com o aumento da qualidade de software e com o uso de ferramentas especializadas.

Segundo Travassos e Kalinowski (2008), os resultados de desempenho de organizações que adotaram o modelo MPS.BR indicam que estas empresas alcançaram maior satisfação dos seus clientes, maior produtividade, capacidade de desenvolver projetos maiores e satisfação com o modelo MR-MPS. Contudo, um número muito pequeno de empresas adotou o modelo na região Norte do Brasil.

Como iniciativa de melhoria da qualidade de seus processos e produtos, o Centro de Tecnologia da Informação e Comunicação (CTIC) da Universidade Federal do Pará (UFPA), em conjunto com o Laboratório de Engenharia de Software (LABES) da UFPA decidiram implantar o modelo tendo como uma das metas o nível $\mathrm{G}$ do MPS.BR. Além de melhorar os processos do CTIC-UFPA, um objetivo importante estava na utilização do ambiente WebAPSEE [Lima Reis e Reis 2007], desenvolvida pelo LABES-UFPA, como ferramenta de apoio a gestão de processos de software. 
Este artigo apresenta essa experiência sob o ponto de vista do uso da ferramenta, focando em lições aprendidas e procurando fornecer subsídios para discussões em torno de tecnologia de processos de software para implantação de MPS.BR.

Na seção 2, a articulação para o investimento em melhoria de processos no CTIC-UFPA é descrita, bem como a estratégia de implementação utilizada na implementação do nível $\mathrm{G}$ do MPS.BR. Na seção 3 é apresentado resumidamente o ambiente WebAPSEE. Na seção 4 é detalhado o foco principal do artigo, indicando como a ferramenta foi utilizada em termos dos dois processos avaliados: Gerência de Projetos e Gerência de Requisitos. Na seção 5, uma análise sobre o uso do ambiente WebAPSEE na implementação do nível G no CTIC-UFPA é apresentada. Na seção 6 são apresentadas as lições aprendidas e na seção 7 as considerações finais do artigo.

\section{Melhoria de Processos de Software no CTIC-UFPA}

O projeto de implementação de Melhoria do Processo de Software no CTIC-UFPA (MPS.CTIC) iniciou em Março de 2007. As atividades foram voltadas para a melhoria da organização e seus processos em geral e não diretamente para um nível em particular do MPS.BR. Alguns projetos piloto foram iniciados ainda em 2007. Em junho de 2008, com a consolidação do processo de desenvolvimento sendo implantado, foram iniciados os projetos que seriam avaliados. A avaliação ocorreu nos meses de novembro e dezembro de 2008 e teve resultado positivo. O projeto como um todo resultou em várias contribuições importantes para a organização, tais como: formação de recursos humanos, capacitação em engenharia de Software, implantação de processos organizacionais de atendimento ao usuário, homologação de ferramentas, suporte da área de redes e capacitação. O principal resultado atingido em 2008 foi a avaliação positiva do nível G para um dos setores do CTIC.

Nesta seção é apresentada a organização CTIC-UFPA com suas particularidades e a estratégia de implementação utilizada para se alcançar melhoria de processo.

\subsection{Organização}

O CTIC é um órgão diretamente ligado à reitoria da UFPA, que tem com missão prover serviços e recursos tecnológicos para a comunidade universitária, em apoio às atividades acadêmicas e administrativas, integrando-se no esforço de oferecer à sociedade uma universidade de excelência. Dessa forma, trata-se de uma unidade estratégica, onde a qualidade da informação e a agilidade para responder as demandas dos usuários são fatores críticos de sucesso.

A estrutura organizacional é composta pelo Diretor, Assessorias, e quatro gerências: Gerência Administrativa, Gerência de Atendimento ao Usuário, Gerência de Suporte e Infra-estrutura, e Gerência de Sistemas de Informação. Além disso, o CTICUFPA conta com 46 funcionários, sendo que 7 possuem mestrado e 9 especialização. Desses funcionários, 14 estão afastados de suas atividades por estarem cedidos a outros órgãos ou cursando Doutorado. Devido à baixa quantidade de funcionários frente às demandas que surgem, a organização conta com mais 39 bolsistas de graduação para auxiliarem na realização das tarefas.

A unidade organizacional onde se concentrou a melhoria em termos de MPS.BR foi a Sub-Gerência Web, subordinada à Gerência de Sistemas de Informação, com 17 colaboradores envolvidos com desenvolvimento de software. 


\subsection{Estratégia de Implementação}

A implantação de processos de software em uma organização é fortemente baseada em conhecimento. Para a implantação de uma iniciativa de melhoria, é fundamental que os responsáveis por executar os processos sejam capacitados. Além disso, uma iniciativa de melhoria é normalmente considerada cara por muitas organizações, pois são necessários recursos significativos durante certo período de tempo. Desta forma, tornase necessário desenvolver estratégias efetivas para implementar processos de software com sucesso [Montoni et al 2008].

Especificamente, no projeto de implementação do nível G do MR-MPS no CTIC-UFPA desenvolvido pelo Grupo de Melhoria de Processos de Software da UFPA foi utilizada a estratégia de implementação de Covre et al (2008). Tal estratégia é baseada no modelo de implantação IDEAL [Mcfeeley 1996], com a definição de cinco estágios (iniciação, diagnóstico, estabelecimento, ação e aprendizagem). Possui procedimentos, templates de documentos, material de treinamento, entre outros voltados para a implementação do MR-MPS. Além disso, essa estratégia tem como ferramental de apoio o ambiente WebAPSEE [Lima et al 2006]. Tanto a metodologia quanto a ferramenta foram desenvolvidos pelo LABES-UFPA.

\section{Ambiente WebAPSEE}

O WebAPSEE permite a modelagem (visual) e execução de processos de software, e tem na flexibilidade de definição e execução (modificações ad-hoc no processo em tempo de execução) o seu principal diferencial. Esse ambiente evoluiu em termos de funcionalidades com o suporte a reutilização de processos [Costa et al 2007] e controle de versão de artefatos [Sales 2007], além de funcionalidades características de ferramentas de gerenciamento de projetos, como visualização do processo com o Gráfico de Gantt, geração de caminho crítico, Estrutura Analítica do Projeto e emissão de relatórios gerenciais, como acompanhamento de atividades com desvio de esforço e prazos, plano de custos e recursos humanos, dentre outros.

Atualmente, o WebAPSEE é implantado com um servidor, que centraliza o repositório de processos e serviços de modelagem, execução, reutilização, gerência de artefatos e acesso a dados, e dois tipos de clientes: o WebAPSEE Manager Console e a WebAPSEE Task Agenda (Figura 1).

No Manager Console, o gerente e o engenheiro de processos podem cadastrar informações organizacionais (sobre pessoas, recursos de apoio, cargos, habilidades, entre outras), modelar processos, acompanhar e interagir (visualmente) com a execução dos processos, alocar pessoas e recursos, gerar relatórios e assim por diante. Já a Task Agenda é o aplicativo cliente utilizado pelos desenvolvedores, através do qual estes podem visualizar suas atividades (com estados e instruções de execução), acessar os artefatos necessários para a execução de cada tarefa e enviar os artefatos produzidos em cada atividade, além de fornecer feedback sobre o andamento de suas tarefas.

\section{Implantação da ferramenta}

Nesta seção o uso da ferramenta é caracterizado com relação aos resultados esperados do nível $G$ do MPS.BR. Cabe ressaltar que os envolvidos no processo utilizaram necessariamente a Task Agenda para registrar o andamento de suas tarefas. Isso foi primordial para a correta utilização dos relatórios de monitoramento da ferramenta. 


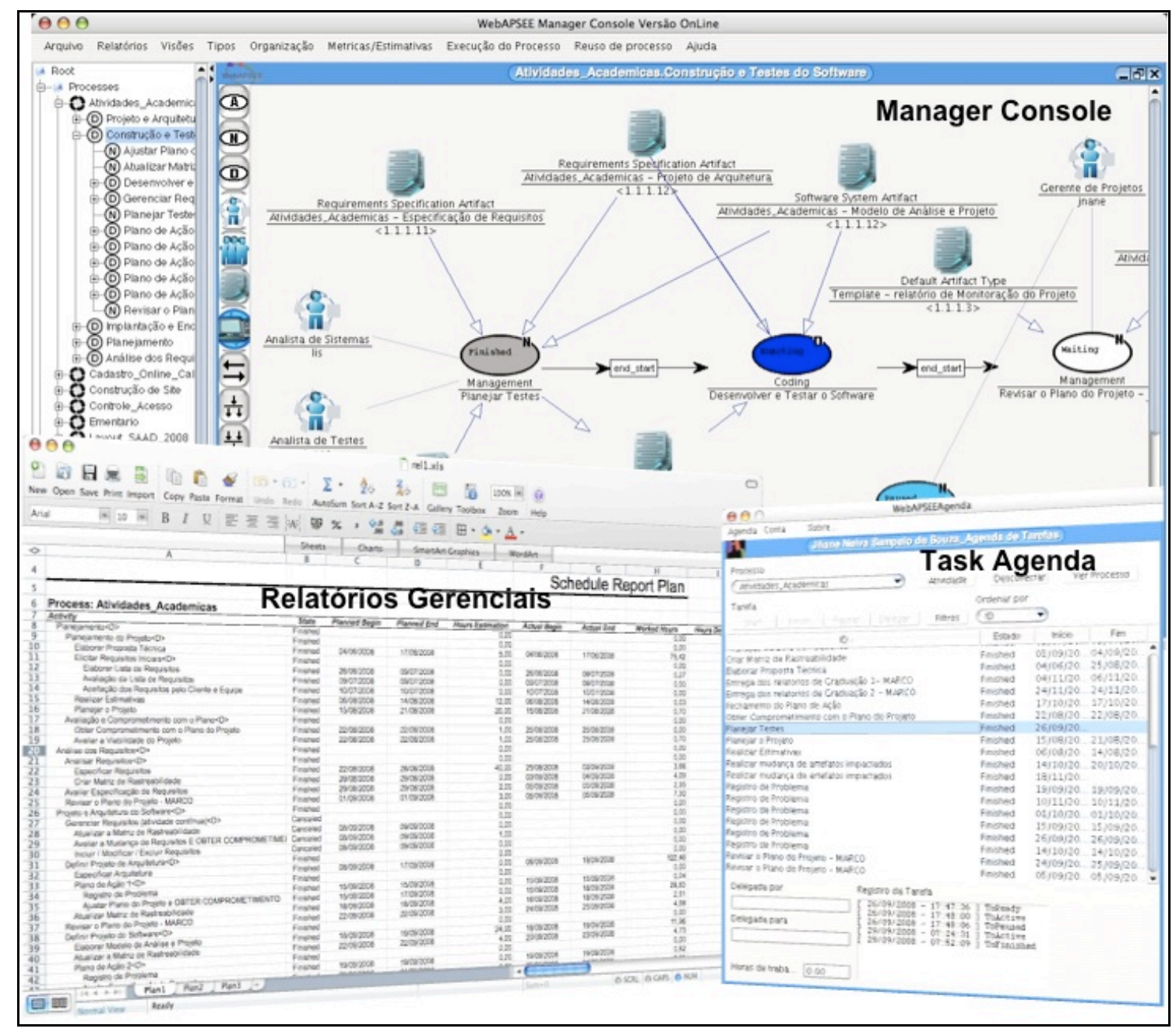

Figura 1. Funcionalidades do ambiente WebAPSEE.

\subsection{Gerência de Projetos}

As principais fontes de evidência para os resultados esperados do processo de Gerência de Projeto (GPR) com a utilização da ferramenta são:

- Modelo do Processo: a ferramenta permitiu a descrição visual do processo sendo executado para um projeto contendo:

- as atividades e seus respectivos estados de execução e a dependência entre elas;

- os artefatos de entrada e saída por atividade, o que permite visualização e manipulação eficiente do plano de gerência de dados e de comunicação;

- as pessoas (e seus cargos) alocadas em atividades, o que permite visualizar e manipular o plano de recursos humanos;

- os recursos de apoio alocados para cada atividade (com quantidades e valores quando pertinente), o que permite visualizar e manipular o plano de recursos de apoio;

- Relatórios gerenciais que evidenciam o planejamento e a situação do projeto para fins de monitoração;

- Métricas coletadas durante a execução do processo para acompanhamento, como, por exemplo, o total de horas trabalhadas por pessoa em cada tarefa; 
- Gerência do acesso controlado aos artefatos do processo.

Atividades específicas de GPR ocorrem em diferentes momentos da execução do processo, em marcos e situações de exceção. Na descrição do processo é possível determinar quando as atividades de planejamento devem ocorrer e quais são os marcos do projeto. Além disso, os planos de ação podem ser modelados como templates de processos [Costa et al 2007] que são instanciados pelo gerente como fragmentos do processo quando uma exceção ocorre na execução do processo.

Como exemplo de implementação de resultados esperados, a gerência de dados é realizada através do Plano de Gerência de Dados: um relatório gerado a partir de informações extraídas do processo instanciado, onde é possível saber quem utiliza, qual artefato, em qual atividade e qual artefato será produzido. Além disso, esses artefatos ficam sob controle da ferramenta através da integração com uma ferramenta de controle de versões (externa, CVS ou SVN), sendo que os usuários terão acesso aos artefatos através da Task Agenda, acessada através de senha com permissão para leitura e escrita de artefatos dependente da situação do processo em andamento.

Outro exemplo de resultado esperado é a monitoração do projeto através do acompanhamento visual da execução do processo e do relatório de acompanhamento de atividades com desvio de esforço e prazo. Em tais casos, relatórios de cronograma com desvio de prazo e geração do gráfico de Gantt do projeto puderam evidenciar atrasos.

\subsection{Gerência de Requisitos}

Da mesma forma que ocorre com o processo GPR, o processo de Gerência de Requisitos (GRE) é modelado de forma integrada ao processo do projeto em andamento. Apesar de GRE ter suas atividades ocorrendo em momentos pré-definidos, existem também atividades que ocorrem em situações que dependem de eventos externos, como uma solicitação de mudança nos requisitos, por exemplo.

$\mathrm{Na}$ ocorrência de uma mudança de requisitos, as atividades do processo de mudança são controladas e acompanhadas na ferramenta da mesma forma que o fluxo normal do processo. Isto significa que os artefatos impactados, as pessoas e recursos alocados durante a mudança também são visualizados como um trecho do processo.

O WebAPSEE ainda não possui uma ferramenta que apóie a rastreabilidade de Requisitos integrada ao ambiente. São utilizados apenas templates para guiar a construção de artefatos como a Matriz de Rastreabilidade, Solicitação de Mudança de Requisitos, Avaliação da Mudança de Requisitos, entre outros. Entretanto, o ambiente permite estabelecer a dependência entre os artefatos através da definição dos relacionamentos de derivação e pertinência entre artefatos, além da ordem em que os artefatos são consumidos e produzidos pelas atividades na descrição visual do processo.

\section{Aspectos coletados sobre o uso do WebAPSEE na implementação do Nível G do MPS.BR}

A partir da experiência de utilização da ferramenta tanto por parte dos usuários, funcionários (Gerentes de Projeto, Analistas, Desenvolvedores) do CTIC-UFPA, quanto dos consultores de implementação, colaboradores do LABES-UFPA, pode-se levantar aspectos relevantes para o processo de implementação de melhoria de processos 
realizado (Ver tabela 1). Tais aspectos não se restringem apenas à Gerência de Projetos e Gerência de Requisitos haja vista o ambiente WebAPSEE apoiar diversas áreas da Gerência de Processo de Software.

Tabela 1 - Pontos Positivos e Oportunidades de Melhoria no Uso do WebAPSEE na implementação do Nível G do MPS.BR no CTIC-UFPA

\begin{tabular}{|c|c|c|}
\hline Tipo & Descrição & Fonte \\
\hline \multirow{7}{*}{ 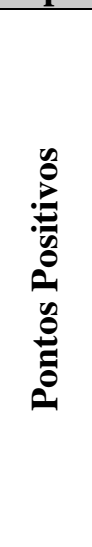 } & $\begin{array}{l}\text { Definição dos processos (tarefas, prazos, agentes, recursos, etc) } \\
\text { através de uma linguagem visual }\end{array}$ & Todos \\
\hline & $\begin{array}{l}\text { Modificação do fluxo de execução dos processos mediante } \\
\text { eventos significativos (atrasos, mudanças nos requisitos, etc.) }\end{array}$ & Gerentes de Projeto \\
\hline & $\begin{array}{l}\text { Facilidade para cadastrar e recuperar as evidências dos processos } \\
\text { de GPR e GRE }\end{array}$ & $\begin{array}{c}\text { Equipe de } \\
\text { Implementação }\end{array}$ \\
\hline & Relatórios gerados automaticamente facilitam a monitoração & Gerentes de Projeto \\
\hline & Controle e armazenamento de artefatos versionados & Todos \\
\hline & Apoio e orientação para execução das tarefas através da agenda & Desenvolvedores \\
\hline & $\begin{array}{l}\text { Acesso as atividades diárias e visualização do modelo de } \\
\text { processo em execução possibilitando maior percepção } \\
\text { (awareness) sobre o processo }\end{array}$ & Desenvolvedores \\
\hline \multirow{3}{*}{ 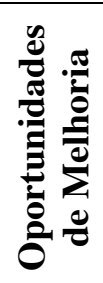 } & Ferramenta para GRE integrada ao ambiente & $\begin{array}{l}\text { Equipe de } \\
\text { Avaliação }\end{array}$ \\
\hline & $\begin{array}{l}\text { Melhoria nas interfaces de cadastro do ambiente quanto à } \\
\text { Usabilidade }\end{array}$ & Gerente de Projetos \\
\hline & $\begin{array}{l}\text { Integração com outras ferramentas de desenvolvimento de } \\
\text { software (IDEs de programação, BugTracking, etc.) }\end{array}$ & Desenvolvedores \\
\hline
\end{tabular}

\section{Discussão sobre as Lições Aprendidas}

Algumas situações ocorridas durante a experiência contribuíram para a melhoria da ferramenta ou para melhoria da estratégia de implementação da equipe. A seguir são apresentados os casos que levaram a algumas lições aprendidas do ponto de vista do uso da ferramenta na estratégia de implementação MPS.BR:

- Como ocorre com PSEEs em geral [Fuggetta 2000], houve a dificuldade inicial em adotar a ferramenta, principalmente considerando que os desenvolvedores teriam sua rotina controlada através da Task Agenda. Foi observada dificuldade em perceber os benefícios da ferramenta principalmente por esta não ajudar diretamente no trabalho da equipe. Como foram executados vários projetos piloto com amplo acompanhamento de pessoal de suporte da ferramenta, foi possível constatar que ao final a equipe "acostumou-se" a usar a agenda. $\mathrm{O}$ apoio da Direção do CTIC e dos gerentes envolvidos foi de grande importância naquele momento inicial da implantação da ferramenta;

- Pudemos constatar que o processo definido (e ajustado durante os projetos) foi seguido à risca com o uso da ferramenta. A execução dos projetos pode inclusive ser auditada através do log de eventos do ambiente. Isso ocorreu porque a execução do processo segue estritamente o que for modelado pelo gerente. Portanto, a equipe não possui apenas um guia de processo para consulta na web, nem tampouco se informa do processo apenas através de reuniões. A ferramenta propicia o seguimento do processo e a pessoa torna-se responsável por fornecer feedback correto, pois do contrário, pode prejudicar o andamento do projeto. 
Para aumentar a responsabilidade, o usuário da agenda tem acesso ao processo e pode navegar no mesmo para compreender o contexto de sua tarefa no todo e as dependências e influências do seu trabalho no trabalho dos outros.

- Apesar da característica de flexibilidade para mudanças durante o processo, notou-se que é necessário que o gerente use essa flexibilidade a favor do projeto e esteja atento aos construtores da linguagem de modelagem para não "engessar" o processo desnecessariamente. Por exemplo, o gerente poderia mudar a dependência no caso de uma atividade que depende da anterior, cujo responsável está ocioso, e que, segundo avaliação dos envolvidos, já poderia começar o trabalho. São ajustes que podem e devem ocorrer para manter o processo sendo executado na ferramenta totalmente sincronizado com o processo real. Foi notado que em alguns momentos a flexibilidade da ferramenta foi sub-utilizada.

- Uma dificuldade de planejamento ocorrida levou ao desenvolvimento de uma funcionalidade nova na ferramenta: era difícil alocar pessoas que trabalham em vários projetos ao mesmo tempo, pois não estava disponível de forma direta a alocação de pessoas em uma data futura. Assim, foi acrescentado ao relatório de recursos humanos uma coluna com a carga de trabalho (número de atividades) de uma pessoa em uma data futura, selecionada pelo usuário.

- Como seria a primeira experiência de avaliação de uma organização que utilizou a ferramenta, a equipe de implementadores optou por criar templates de documentos que traziam algumas redundâncias em relação ao conteúdo da ferramenta. Tais redundâncias faziam com que o gerente gerasse um relatório no WebAPSEE e copiasse o conteúdo do mesmo para os templates fornecidos. Isso gerou uma sensação maior de segurança para efeito de avaliação, mas diminuiu a agilidade pretendida com o uso da ferramenta. Nas próximas experiências pretende-se usar mais as evidências fornecidas pela ferramenta para efeito de avaliação.

- Além do planejamento das monitorações nos marcos do projeto, a ferramenta Manager Console mostra ao gerente instantaneamente a situação atual de seus projetos. Isso agilizou bastante a tomada de decisões e fez com que os gerentes passassem a criar planos de ação para solução de eventuais problemas com base na monitoração constante da ferramenta, obtida a partir do feedback da equipe. Em vários casos, o marco atingido serviu apenas pra checar e documentar para a próxima fase se todos os problemas estavam resolvidos. Ou seja, o gerente não necessitava reunir-se com tanta freqüência para ficar ciente dos problemas.

- Como a ferramenta é integrada ao controle de versões de artefatos, uma funcionalidade simples ajudou bastante na coleta de evidências para avaliação. Cada vez que um artefato era submetido como produção de uma atividade, era gravada a versão e adicionalmente era registrada a atividade que gerou $\mathrm{o}$ artefato, a pessoa que fez upload além da data e hora. Com isso, obter a versão do plano de projeto no marco da fase de análise de requisitos é uma tarefa simples.

\section{Considerações Finais}

Este artigo descreveu a implementação do MR-MPS em uma organização paraense utilizando o ambiente WebAPSEE. A partir dessa experiência foi possível analisar as 
situações ocorridas e transformá-las em lições aprendidas tanto para desenvolvimento da ferramenta quanto para estratégia de implementação.

A empresa foi avaliada em dezembro de 2008 e obteve o nível pretendido com o uso do ambiente WebAPSEE reportado como ponto forte, o que comprova o sucesso da abordagem. Cabe ressaltar que a equipe do CTIC-UFPA não tinha experiência anterior em nenhuma ferramenta correlata. Portanto, não foi possível obter da organização nenhum comparativo com outras situações e ferramentas que pudessem trazer lições aprendidas ou novas demandas de funcionalidades. Um aspecto resultante disso é que não foram registrados pontos negativos no uso da ferramenta.

As lições aprendidas com a experiência são importantes para melhorar a estratégia de implementação adotada e estão gerando novas demandas de funcionalidades para a ferramenta que atendam melhor o nível $\mathrm{G}$ e também os níveis superiores.

\section{Referências}

Costa, A.; Sales, E.; Lima Reis, C.; Reis, R. (2007) “Apoio a Reutilização de Processos de Software através de Templates e Versões", In: 6 Simpósio Brasileiro de Qualidade de Software (SBQS'07). Porto de Galinhas, PE, Brasil.

Covre, V.; Lima Reis, C.; Favero, E. (2008) "Metodologia para Implementação do MPS.BR Utilizando o Ambiente WebAPSEE”, In: $7^{\circ}$ Simpósio Brasileiro de Qualidade de Software (SBQS'08). Florianópolis, SC, Brasil.

Fuggetta Alfonso. (2000) "Software Process: a roadmap", In: Conference on the future of Software engineering (ICSE '00). Limerick, Ireland.

Lima, A. et al. (2006) "Gerência Flexível de Processos de Software com o Ambiente WebAPSEE”. In: $19^{\circ}$ Simpósio Brasileiro de Engenharia de Software (SBES'06) Sessão de Ferramentas. Florianópolis, SC, Brasil.

Lima Reis, C. A.; Reis, R. Q. (2007) Laboratório de Engenharia de Software e Inteligência Artificial: Construção do ambiente WebAPSEE. In ProQuality (UFLA), v. 3, p. 43-48.

Mcfeeley, B. (1996), IDEAL ${ }^{\text {SM. }}$ A User's Guide for Software Process Improvement. Carnegie Melon University, Software Engineering Institute, Pittsburgh.

Montoni, M.; Santos, G.; Katsurayama, A.; Cabral, R.; Figueiredo, S.; Natali, A. (2008) "Aplicação da Estratégia SPI-KM para Apoiar a Implementação do MPS.BR Níveis G e F em Pequenas e Médias Empresas do Rio de Janeiro" ", In: $7^{\circ}$ Simpósio Brasileiro de Qualidade de Software (SBQS'08). Florianópolis, SC, Brasil.

Softex (2007) "MPS.BR - Melhoria de Processo do Software Brasileiro - Guia Geral", versão 1.2 , junho.

Sales, E.; Lima Reis, C.; Lima, A. (2007) "Gestão de Configuração integrada a Gerência de Processos de Software no Ambiente WebAPSEE”, In: $33^{\text {a }}$ Conferência Latino Americana de Informática (CLEI'07). San José, Costa Rica.

Travassos, G.; Kalinowski, M. (2008) "iMPS: resultados de desempenho de organizações que adotaram o modelo MPS", Relatório Técnico. Campinas, SP, Brasil. 